\title{
The source matters: Agreement and accuracy of race and ethnicity codes in Medicare administrative and assessment data
}

\author{
Olga F. Jarrín \\ Irina B. Grafova \\ Abner N. Nyandege \\ XinQi Dong \\ Rutgers, The State University of New Jersey \\ Correspondence: olga.jarrin@rutgers.edu \\ Funding information: AHRQ R00-HS022406
}

\begin{abstract}
Background: Errors in racial and ethnic classification of Medicare beneficiaries limit health services research on minority health and health disparities among priority populations, including American Indians and Alaskan Natives.

Objective: To compare the agreement and accuracy of three sources of race and ethnicity information contained in the Medicare data warehouse: 1) the Enrollment Database (EDB) which originate from Social Security data; 2 ) the Research Triangle Institute (RTI) imputed data based on name and geography; and 3) self-reported race and ethnicity data collected during routine home health care assessments as part of the Outcome and Assessment Information Set (OASIS). Subjects: Medicare beneficiaries over the age of 18 who received home health care in $2015(\mathrm{~N}=$ 4,243,090).

Measures: Percent agreement, sensitivity, specificity, positive predictive value, and Cohen's kappa coefficient.

Results: Compared to self-reported race/ethnicity data from OASIS, the RTI race code is more accurate than the EDB race code. Non-Hispanic whites and blacks were correctly classified by the RTI race code with 97\% accuracy. However, more than half of American Indians/Alaskan Natives, one-fourth of Asian American/Pacific Islanders, and nearly one-tenth of Hispanics were misclassified by the RTI race code. Misclassification of race/ethnicity occurred less often for men, compared to women.

Discussion: These findings highlight the strengths and limitations of using race/ethnicity classifications contained in Medicare administrative data. Health services and policy researchers should consider using self-identified race/ethnicity information to augment administrative data sources. This is especially important for research that aims to include Asian Americans/Pacific Islanders and American Indians/Alaskan Natives.
\end{abstract}




\section{INTRODUCTION}

Improving minority health and reducing health dispartities is a national priority. ${ }^{1,2}$ Recent attention has been placed on addressing confounding of observational data and the use of sophisticated causal modeling methods in health disparities research. ${ }^{3}$ However, monitoring and reducing disparities requires accurate data on race and ethnicity that is not consistently available. ${ }^{4-6}$ Administrative data sources of race/ethnicity data are limited with regards to completeness and accuracy, making self-reported data the preferred source and gold standard. ${ }^{7}$ Despite this, even when self-reported race/ethnicity data is available, an administrative data source is frequently used in research on disparities in healthcare quality and outcomes. ${ }^{8-10}$ The completeness and accuracy of race/ethnicty data is especially problematic for Asian Americans and Pacific Islanders (AAPI), as well as for American Indians and Alaskan Natives (AIAN). ${ }^{11-13}$ As a result, incomplete and inaccurate race/ethnicity data limit our understanding of the sources of disparities in healthcare access, quality, and outcomes as well as evaluation of changes in minority health over time.

Administrative data, including insurance plan enrollment and demographic information, is contained in the Medicare Beneficiary Summary File (MBSF). The MBSF contains two separate race variables. The first is from the Medicare enrollment database (EDB), and originates from Social Security Administration records. The EDB race variable is known to severely undercount Hispanics, Asian Americans/Pacific Islanders, and American Indians/Alaskan Natives. ${ }^{14}$ Due to these limitations, analyses using race/ethnicity data from the enrollment file (EDB) are generally restricted to the identification of differences between black and white patient populations. ${ }^{9,15}$ The second race variable was created a decade ago by researchers at the Research Triangle Institute (RTI) to improve classification of Hispanics and Asians/Pacific Islanders. ${ }^{16,17}$ The RTI race imputation algorithm utilizes lists of Hispanic and Asian/Pacific Islander names from the U.S. Census, and simple geography (residence in Puerto Rico or Hawaii) to improve on the EDB race code. ${ }^{16}$ The RTI race variable is used by the Centers for Medicare \& Medicaid Services' in reports on health disparities in the Medicare population and in studies which include focus on Hispanic and Asian/Pacific Islander populations. ${ }^{18,19}$

In contrast to administrative data sources, national surveys of Medicare beneficiaries include self-reported race and ethnicity. Examples of survey datasets that contain self-reported race/ethnicity include the Medical Expenditure Panel Survey (MEPS), the Medicare Current Beneficiary Survey (MCBS), and the Health and Retirement Survey (HRS). Additionally, the Consumer Assessment of Healthcare Providers and Systems (CAHPS) patient experience datasets contain self-reported race ethnicity data. Finally, self-reported race/ethnicity data is collected as part of post-acute and long-term care assessments including the Outcome and Assessment Information Set (OASIS) used in home health care, the Minimum Dataset (MDS) used in nursing homes, the Inpatient Rehabilitation Facility-Patient Assessment Instrument (IRFPAI), and the Medicare Health Outcomes Survey (HOS) used in Programs of All-Inclusive Care of the Elderly and with a random sample of Medicare Advantage plan subscribers.

While patient experience survey data (CAHPS) has been used to validate race/ethnicity variables contained in administrative sources, the use of self-reported race/ethnicity data collected as a routine part of healthcare delivery has received less attention. The main objective of this analysis 
is to compare the agreement and accuracy of three sources of race and ethnicity information contained in the Medicare data warehouse: 1) the Enrollment Database (EDB) race variable which originates from Social Security Administration data; 2) the Research Triangle Institute (RTI) race variable imputed from name and geography; and 3) self-reported race and ethnicity data collected by Registered Nurses and Physical Therapists during routine home health care assessments as part of the Outcome and Assessment Information Set (OASIS). ${ }^{20}$ For added context, the accuracy and agreement measures are stratified by sex, patterns of misclassification errors are explored, and we compare our findings with earlier studies using survey data as the gold standard.

\section{METHODS}

\section{Data Source and Patient Population}

Two sources of race/ethnicity data for Medicare beneficiaries were combined for calendar year 2015: The Medicare Beneficiary Summary File (MBSF) containing the Enrollment Database (EDB) and Research Triangle Institute (RTI) race variables, and the Outcome and Assessment Information Set (OASIS) containing demographic data on all home health care patients. Demographic information on the OASIS is collected during the initial home health care visit by a registered nurse or licensed physical therapist. Race/ethnicity data is obtained by self-report (a caregiver may answer if the patient is unable) and allows for multiple answers to be recorded. The vast majority (99.7\%) of home health beneficiaries had only a single race/ethnicity recorded, and we restricted our study to this population.

The study population included all Medicare beneficiaries, 18 years and older, who received home health care, and self-identified with only one race/ethnicity on the home health. Details of the 11,720 people $(0.3 \%$ of study population) who identified with two or more racial/ethnic groups are included for the interested reader as a brief Appendix. The final study population consisted of 4,231,370 adult Medicare beneficiaries who received home health care in 2015. The study was approved by the Institutional Review Board of Rutgers, The State University of New Jersey.

\section{Statistical Analyses}

We performed three sets of analyses. All analyses were performed using SAS statistical software (version 9.4). First, we used the self-reported OASIS race as the validation standard to assess the agreement and accuracy of the EDB and RTI race variables, contained in linked Medicare administrative data. Datasets were linked at the patient level using a unique beneficiary identification code assigned for this purpose by CMS. Analyses of sensitivity, specificity, positive predictive value (PPV), and Cohen's kappa coefficient were calculated for the full sample and for each sex separately (Table 1). Sensitivity indicates the proportion of beneficiaries identified in the OASIS data in a specific race/ethnicity category who are correctly identified on

the MBSF. Specificity is the probability that someone who is truly not in a specific race/ethnicity category in the MBSF will be classified as not affiliated with that race/ethnicity in the OASIS data. Positive predictive value is the proportion of beneficiaries identified by the MBSF in a specific race/ethnicity category who are self-identified in the same category on the OASIS data. 
The Cohen kappa coefficient ${ }^{21}$ is a widely used measure of interrater reliability, ranging from 1 (complete agreement) to -1 (complete disagreement). As a point of reference, Landis and Koch suggest excellent agreement is indicated by a kappa coefficient greater than $0.81 .^{22}$

In the second set of analyses, the pattern of race/ethnicity misclassification are shown for both EDB and RTI race variables (Table 2). These analyses augment the accuracy and agreement statistics and highlight the more nuanced differences between the pattern of racial/ethnic misclassification errors in the EDB and RTI race variables.

In the third set of analyses, differences in race/ethnicity categorization of RTI compared to OASIS race/ethnicity are compared for a subset of beneficiaries with dementia or diabetes (Table 3). We determined dementia or diabetes diagnosis status for our subset study population from the Medicare Beneficiary Summary File (MBSF) chronic conditions warehouse flags. This analysis highlights one aspect of race/ethnicity variable choice on study design and the resulting differences in frequency and prevalence of chronic disease burden within subpopulations.

\section{RESULTS}

\section{Agreement and accuracy of Enrollment Data Base (EDB) and Research Triangle Institute (RTI) race variables with self-reported race/ethnicity from OASIS.}

Both the EDB and RTI race variables have mutually exclusive categories, meaning that a person who is categorized as white or black is considered to be non-Hispanic. In our analyses, using OASIS race as the validation standard, the specificity of EDB race for non-Hispanic whites was 79.5 compared to 95.5 for the imputed RTI race variable (Table 1). In other words, there is one in five chance that someone who is not white will be misclassified as white using the EDB race variable, but only a $4.5 \%$ chance of misclassification as white when using the RTI race variable. Among people who self-identified as non-Hispanic black, both the EDB and RTI race variables perform similarly, with high specificity (99.2-99.4) and high sensitivity (96.6-97.0).

As the EDB race variable originates from social security records, an Afro-Cuban naturalized U.S. citizen would be classified as Hispanic, based on country of birth, and this classification would be retained in the (imputed) RTI race variable. In our analyses, for people who selfidentified as Hispanic, the original EDB variable had high specificity (99.8) but low sensitivity (36.2). In contrast, the RTI race variable had both high specificity (98.7) and good sensitivity (90.8), undercounting fewer than one in ten Hispanics.

Among people who self-identified as Asian, Hawaiian Native, or other Pacific Islander (AAPI), specificity of both the EDB and RTI race variables was high (99.6-99.7). However, the RTI race variable had better sensitivity (74.7) compared to the EDB race variable (65.0). Meaning, one in four people who self-identified as AAPI were undercounted using the RTI race variable, compared to the EDB race variable which undercounted one in three Asian American/Pacific Islanders.

Among people who self-identified as American Indian or Alaskan Native (AIAN) the specificity of the EDB and RTI race variables was high (99.8), however the sensitivity was low (43\%). The 
EDB classification of AIANs using tribal membership records resulted in fewer than half of AIANs were correctly classified in Medicare administrative race/ethnicity data (Table 1).

\section{Sex differences in accuracy and agreement of race/ethnicity variables}

The EDB race variable, originating from Social Security Administration records, is slightly more accurate for women compared to men among Hispanics $(k=0.50$ vs. 0.49$)$, AAPIs $(k=0.72$ vs. $0.69)$, and whites ( $k=0.82$ vs. 0.80$)$ (Table 1). In contrast the RTI race variable, imputed from U.S. Census name lists and residence in Hawaii or Puerto Rico, is less accurate for women compared to men among Hispanics $(k=0.85$ vs. 0.89$)$ and AAPIs $(k=0.76$ vs. 0.78$)$.

\section{Patterns of over-classification and misclassification by race/ethnicity variables}

The pattern of misclassification errors in the EDB and RTI race variables compared to selfreported race/ethnicity from the OASIS dataset are shown in Table 2. Using the original EDB race variable 190,434 people were misclassified as non-Hispanic white, with the majority (88\%) self-identifying as Hispanic. In contrast, the RTI race variable mistakenly classifies a much smaller fraction of minorities as being non-Hispanic white. However, using the RTI race variable non-Hispanic whites are misassigned as Hispanic more than five times as often compared to the original EDB race variable, accounting for $78 \%$ of people misassigned to Hispanic ethnicity. Similarly, non-Hispanic whites comprise $80 \%$ of people misassigned by the RTI race variable as black, 77\% of people misassigned as Asian/Pacific Islander, and 84\% of people misassigned as American Indian/Alaskan Native (Table 2).

\section{Dementia and diabetes frequency and prevalence by race/ethnicity variables}

To illustrate the potential impact of race/ethnicity misclassification on estimated size of health disparities and disease prevalence we calculated the number of beneficiaries with dementia and diabetes using each of the three race/ethnicity variables. When comparing the numbers of people with a diagnosis of dementia or diabetes the largest net differences were among the Hispanics, Asians/Pacific Islanders, and American Indians/Alaskan Natives (Table 3). The net difference is important for study designs that draw their sampling frame from administrative data sources.

Using the RTI race variable (compared to OASIS) resulted in an overestimation of the number of Hispanics with dementia by a net difference of 4,283 (4.8\%) and diabetes by a net difference of 10,477 (5.4\%) (see Table 3). In contrast, the EDB race variable underestimated the number of Hispanics with dementia by a net difference of 48,407 (-54.8\%) and diabetes by a net difference of $114,003(-59.0 \%)$. However, the EDB race variable also produced falsely high estimates of the prevalence of dementia (34.1\%) and diabetes (67.9\%) in Hispanics (see Figure 1). The RTI and OASIS race variables produced similar estimates of the prevalence of dementia $(29.0 \%-29.6 \%)$ and diabetes (63.9\%-64.9\%) among Hispanics.

Among AAPIs, the number of people with dementia was underestimated by a net difference of $1,853(-6.4 \%)$ using the RTI race variable and by 6,032 (-21.1\%) using the EDB race variable. The pattern was similar for diabetes in AAPIs, which was underestimated by a net difference of 4,391 (-8.2\%) using the RTI race variable, and 12,113 (-22.6\%) using the EDB race variable 
(Table 3). When the prevalence of dementia and diabetes were calculated for AAPIs using each of the race/ethnicity variables the pattern was similar to that seen for Hispanics, with EDB race overestimating chronic disease burden (Figure 1). Using the RTI and OASIS variables the prevalence of dementia among Asians/Pacific Islanders was 32.1\%-32.6\%, and 34.1\% using EDB race. For diabetes, the prevalence among AAPIs was 59.9\%-60.2\% using the RTI and OASIS race variables, and $62.7 \%$ using EDB race.

\section{DISCUSSION}

Consistent with prior studies, we found the race variables contained in Medicare administrative data undercount Hispanics, Asians/Pacific Islanders, and American Indians/Alaskan Natives. ${ }^{17,23,24}$ While advances have been made in Medicare Bayesian Improved Surname and Geocoding (MBISG) algorithm used to calculate racial and ethnic differences in Healthcare Effectiveness Data and Information Set (HEDIS) measures, ${ }^{25-27}$ the accuracy and agreement statistics of the MBISG 2.0 have not, to our knowledge, been published. As a benchmark for our findings we briefly summarize published accuracy and agreement statistics for EDB and RTI race/ethnicity variables using different 'gold standard' survey data sources in Table 4. From a methodological standpoint, the choice of race/ethnicity data source is essential at the study design stage for health disparities research. The impact of race/ethnicity variable selection on estimates of disease prevalence is of special concern, as we found in the case of dementia prevalence among Hispanics shown in Figure 1. When using the EDB race variable, the prevalence of dementia among Hispanics is $18 \%$ higher compared to when the RTI race variable is used, with an absolute difference of just over 5 percentage points (see Figure 1). A smaller difference (1.5 percentage points) is seen for AAPIs, with virtually no difference for nonHispanic whites, blacks, and American Indians/Alaskan Natives (AIANs). Compared to the EDB race variable, if the RTI variable was a "race-specific" anti-dementia drug for Hispanics it would be a blockbuster.

For AAPI populations, our study findings have additional significance. Asian Americans/Pacific Islanders are the fastest growing population in the U.S., while being the most heterogeneous. Certain AAPI subgroups, such as Filipinos, may be more prone to misclassification using surname based imputation methods due to the long history of Spanish colonization in the Philippines. Similarly, the Republic of China (Taiwan) was colonized by the Dutch and Spanish; India was colonized by the Portuguese, Dutch, and British; and Vietnam, Laos, and Cambodia were colonized by the French. In addition, interracial/intercultural marriages frequently result in women changing their last name to that of their husband's family, such that a woman who marries a Filipino-American man might be classified as Hispanic using name-based race algorithms.

While the self-reported race/ethnicity data should always be the first choice, we found the RTI race variable to be very accurate for identifying Hispanics $(k=0.89$ for males; $k=0.85$ for females), and non-Hispanic whites $(k=0.90)$ or blacks $(k=0.96)$ of either sex (Table 1). For more granular analyses, and especially research that aims to disentangle race/ethnicity and socioeconomic status, a higher level of accuracy may be desired. Researchers who are working with linked administrative and assessment datasets should report racial/ethnic differences based on the self-reported race variable. Reviewers and journal editors should question the source of 
race/ethnicity data and critically examine the rationale for research which uses the EDB race variable, especially when reporting on black/white disparities. Additionally, future advances in race/ethnicity imputation algorithms should augment and include all self-reported race/ethnicity data from both survey (MCBS, HOS, CAHPS) and assessment (OASIS, MDS) data sources.

This study has several limitations. First, the study population consisted only of Medicare beneficiaries who utilized home health care in calendar year 2015. Compared to all Medicare beneficiaries, people who utilize home health care are older and have a higher burden of chronic disease. Second, blacks are slightly overrepresented in the home health care population compared to the full Medicare population (estimated with the RTI race variable). Third, some older adults, especially AAPIs and Hispanics, may retire or seek supportive care outside of the U.S., limiting their access and use of the Medicare home health care benefit, and the generalizability of findings for these individuals. Finally, AIANs who live on tribal reservations may be underrepresented, in contrast to people who identify as American Indians but are not tribal members.

In conclusion, administrative datasets are commonly used in reports and studies of minority health and health disparities. Our study highlights the potential for bias and error introduced during the selection of race/ethnicity data source. Our work confirms the advantages of using the RTI race variable compared to EDB race variable. We also show that further reductions in error and bias can be gained by using self-reported race/ethnicity contained in assessment datasets. These findings have important implications for the design of future studies and the interpretation of prior published research. Future work to improve imputation algorithms for race/ethnicity should be used to supplement existing sources of self-reported race/ethnicity data. 


\section{References}

1. Perez-Stable EJ, Collins FS. Science visioning in minority health and health disparities. Am J Pub Health 2019;109:S5.

2. Department of Health and Human Services. HHS Action Plan to Reduce Racial and Ethnic Health Disparities: A Nation Free of Disparities in Health and Health Care. Washington, DC; 2015.

3. Jeffries N, Zaslavsky AM, Diez Roux AV, et al. Methodological approaches to understanding causes of health disparities. Am J Pub Health 2019;109:S28-S33.

4. Duran DG, Perez-Stable EJ. Science visioning to advance the next generation of health disparities research. Am J Pub Health 2019;109:S11-S3.

5. Bilheimer LT, Sisk JE. Collecting adequate data on racial and ethnic disparities in health: The challenges continue. Health Affairs 2008;27:383-91.

6. Ng JH, Ye F, Ward LM, Haffer SC, Scholle SH. Data on race, ethnicity, and language largely incomplete for managed care plan members. Health Aff (Millwood) 2017;36:548-52.

7. Executive Office of the President Office of Management and Budget, Office of Information and Regulatory Affairs. Revisions to the standards for the classification of federal data on race and ethnicity. Washington, DC: Federal Register; 1997:58782-90.

8. Figueroa JF, Zhou X, Jha AK. Characteristics and spending patterns of persistently high-cost Medicare patients. Health Aff (Millwood) 2019;38:107-14.

9. Joynt Maddox KE, Chen LM, Zuckerman R, Epstein AM. Association between race, neighborhood, and Medicaid enrollment and outcomes in Medicare home health care. J Am Geriatr Soc 2018;66:239-46.

10. Belanger E, Silver B, Meyers DJ, et al. A retrospective study of administrative data to identify high-need Medicare beneficiaries at risk of dying and being hospitalized. J Gen Intern Med 2019.

11. Bierman AS, Lurie N, Collins KS, Eisenberg JM. Addressing racial and ethnic barriers to effective health care: The need for better data. Health Affairs 2002;21:91-102.

12. Thomson GE, Mitchell F, Willimams MB, eds. Examining the Health Disparities Research Plan of the National Institutes of Health: Unfinished Business. Washington, DC: National Academies Press; 2006.

13. Race, Ethnicity, and Language Data: Standardization for Health Care Quality Improvement. Washington, DC: The National Academies Press; 2009.

14. Filice CE, Joynt KE. Examining race and ethnicity information in Medicare administrative data. Med Care 2017;55:e170-e6.

15. Li Y, Cai X, Glance LG. Disparities in 30-day rehospitalization rates among Medicare skilled nursing facility residents by race and site of care. Med Care 2015;53:1058-65.

16. Eicheldinger C, Bonito A. More accurate racial and ethnic codes for Medicare administrative data. Health Care Financ Rev 2008;29:27-42.

17. Bonito AJ BC, Eicheldinger C, Carpenter L. Creation of new race-ethnicity codes and socioeconomic status (SES) indicators for Medicare beneficiaries. Final report, sub-task 2. Rockville, MD: Agency for Healthcare Research and Quality; 2008 January 2008. Report No.: AHRQ Publication No. 08-0029-EF.

18. Lim E, Gandhi K, Davis J, Chen JJ. Prevalence of chronic conditions and multimorbidities in a geographically defined geriatric population with diverse races and ethnicities. J Aging Health 2018;30:421-44. 
19. Centers for Medicare \& Medicaid Services Office of Minority Health. The Mapping Medicare Disparities Tool Technical Documentation, Version 6.0. August 31, 2018. https://www.cms.gov/About-CMS/Agency-Information/OMH/Downloads/MappingTechnical-Documentation.pdf

20. O'Connor M, Davitt JK. The Outcome and Assessment Information Set (OASIS): a review of validity and reliability. Home Health Care Serv Q 2012;31:267-301.

21. Cohen J. A coefficient of agreement for nominal scales. Educational and Psychological Measurement 1960;20:37-46.

22. Landis JR, Koch GG. The measurement of observer agreement for categorical data. Biometrics 1977;33:159-74.

23. Waldo DR. Accuracy and bias of race/ethnicity codes in the Medicare Enrollment Database. Health Care Financing Review 2004;26:61-72.

24. Zaslavsky AM, Ayanian JZ, Zaborski LB. The validity of race and ethnicity in enrollment data for Medicare beneficiaries. Health Serv Res 2012;47:1300-21.

25. Haas A, Elliott MN, Dembosky JW, et al. Imputation of race/ethnicity to enable measurement of HEDIS performance by race/ethnicity. Health Serv Res 2019;54:13-

26. Dembosky JW, Haviland AM, Haas A, et al. Indirect estimation of race/ethnicity for survey respondents who do not report race/ethnicity. Medical Care 2018.

27. Bykov K, Franklin JM, Toscano M, et al. Evaluating cardiovascular health disparities using estimated race/ethnicity: A validation study. Med Care 2015;53:1050-7. 
Dementia Prevalence by Race Variable

$\square$ OASIS RTI EDB

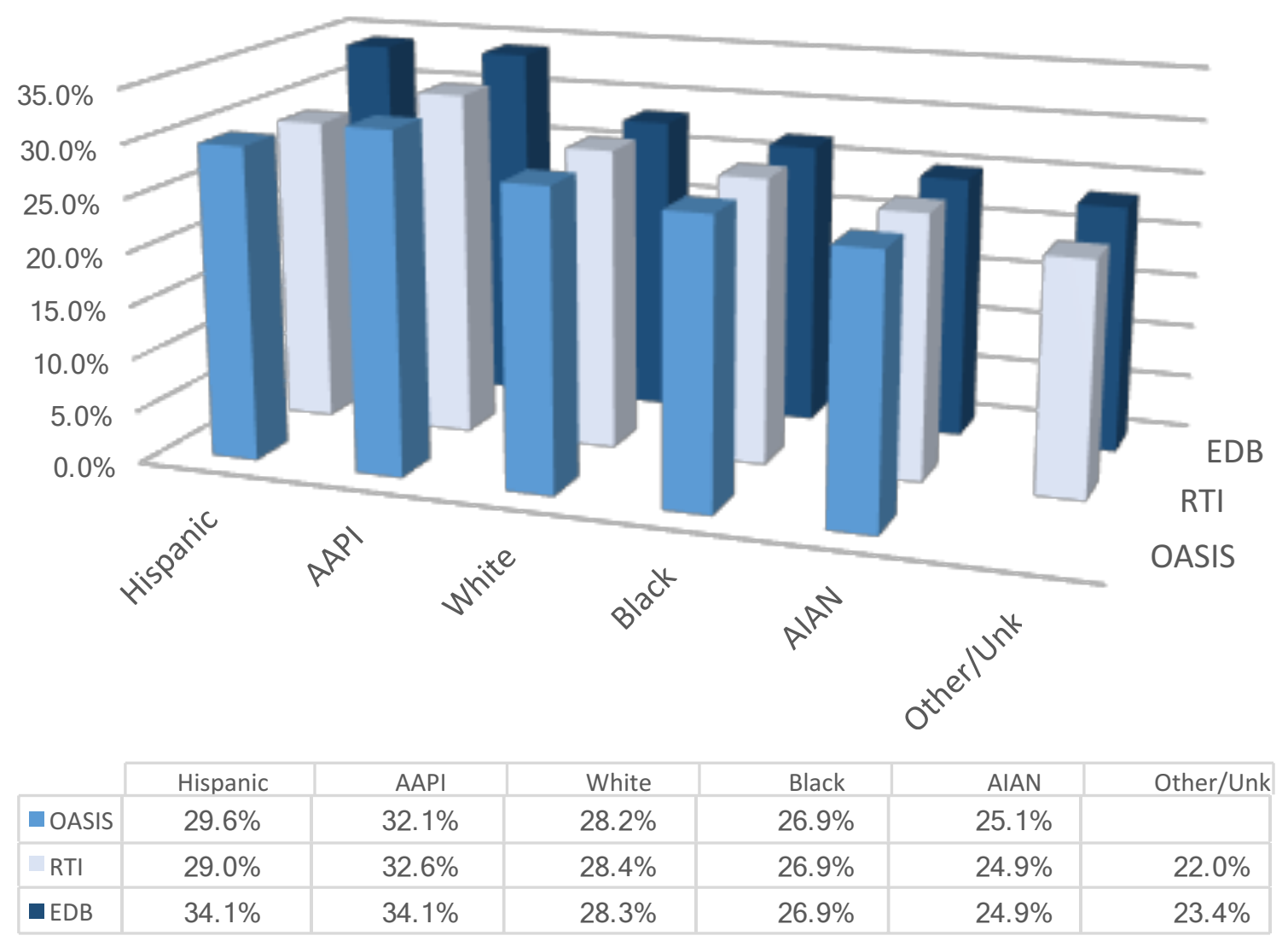

Diabetes Prevalence by Race Variable

$\square$ OASIS RTI EDB

FIGURE 1 Prevalence of Chronic Disease within a Defined Population, Conditional on Choice of Race/Ethnicity Variable 
TABLE 1 Accuracy and Agreement of the Enrollment Data Base (EDB) and RTI Race/Ethnic Classifications Using the Home Health Care Outcome and Assessment Information Set (OASIS) for Comparison: 2015

\begin{tabular}{|c|c|c|c|c|c|c|c|c|}
\hline $\begin{array}{l}\text { Reference }= \\
\text { OASIS race }\end{array}$ & $\begin{array}{r}\text { True } \\
\text { positive }\end{array}$ & $\begin{array}{r}\text { False } \\
\text { negative }\end{array}$ & False positive & True negative & Sensitivity & Specificity & PPV & Kappa \\
\hline \multicolumn{9}{|c|}{ EDB race variable } \\
\hline Hispanic & 107,753 & 190,052 & 9,079 & $3,924,486$ & 36.2 & 99.8 & 92.2 & 0.50 \\
\hline Male & 43,047 & 77,852 & 3,406 & $1,525,418$ & 35.6 & 99.8 & 92.7 & 0.49 \\
\hline Female & 64,706 & 112,200 & 5,673 & $2,399,068$ & 36.6 & 99.8 & 91.9 & 0.50 \\
\hline$A A P I$ & 52,616 & 28,364 & 13,683 & $4,136,707$ & 65.0 & 99.7 & 79.3 & 0.71 \\
\hline Male & 20,491 & 11,705 & 5,836 & $1,611,691$ & 63.6 & 99.6 & 77.8 & 0.69 \\
\hline Female & 32,125 & 16,659 & 7,847 & $2,525,016$ & 65.8 & 99.7 & 80.4 & 0.72 \\
\hline White & $3,229,553$ & 69,983 & 190,434 & 741,400 & 97.9 & 79.5 & 94.4 & 0.81 \\
\hline Male & $1,259,106$ & 32,469 & 76,300 & 281,848 & 97.5 & 78.7 & 94.3 & 0.80 \\
\hline Female & $1,970,447$ & 37,514 & 114,134 & 459,552 & 98.1 & 80.1 & 94.5 & 0.82 \\
\hline Black & 513,158 & 15,630 & 28,042 & $3,674,540$ & 97.0 & 99.2 & 94.8 & 0.95 \\
\hline Male & 188,826 & 6,204 & 10,786 & $1,443,907$ & 96.8 & 99.3 & 94.6 & 0.95 \\
\hline Female & 324,332 & 9,426 & 17,256 & $2,230,663$ & 97.2 & 99.2 & 95.0 & 0.95 \\
\hline AIAN & 6,892 & 9,080 & 8,061 & $4,207,337$ & 43.1 & 99.8 & 46.1 & 0.44 \\
\hline Male & 2,846 & 3,700 & 3,018 & $1,640,159$ & 43.5 & 99.8 & 48.5 & 0.46 \\
\hline Female & 4,046 & 5,380 & 5,043 & $2,567,178$ & 42.9 & 99.8 & 44.5 & 0.43 \\
\hline \multicolumn{9}{|c|}{ RTI race variable } \\
\hline Hispanic & 270,370 & 27,435 & 48,592 & $3,884,973$ & 90.8 & 98.7 & 84.8 & 0.87 \\
\hline Male & 111,779 & 9,120 & 16,089 & $1,512,735$ & 92.5 & 98.9 & 87.4 & 0.89 \\
\hline Female & 158,591 & 18,315 & 32,503 & $2,372,238$ & 89.6 & 98.6 & 83.0 & 0.85 \\
\hline$A A P I$ & 66,696 & 22,573 & 15,594 & $4,126,507$ & 74.7 & 99.6 & 81.0 & 0.77 \\
\hline Male & 27,207 & 8,466 & 6,086 & $1,607,964$ & 76.3 & 99.6 & 81.7 & 0.78 \\
\hline Female & 39,489 & 14,107 & 9,508 & $2,518,543$ & 73.7 & 99.6 & 80.6 & 0.76 \\
\hline White & $3,196,880$ & 102,656 & 41,878 & 889,956 & 96.9 & 95.5 & 98.7 & 0.90 \\
\hline Male & $1,249,568$ & 42,007 & 14,562 & 343,586 & 96.7 & 95.9 & 98.8 & 0.90 \\
\hline Female & $1,947,312$ & 60,649 & 27,316 & 546,370 & 97.0 & 95.2 & 98.6 & 0.90 \\
\hline Black & 511,064 & 17,724 & 21,699 & $3,680,883$ & 96.6 & 99.4 & 95.9 & 0.96 \\
\hline Male & 188,307 & 6,723 & 7,953 & $1,446,740$ & 96.5 & 99.4 & 95.9 & 0.96 \\
\hline Female & 322,757 & 11,001 & 13,746 & $2,234,143$ & 96.7 & 99.4 & 95.9 & 0.96 \\
\hline$A I A N$ & 6,872 & 9,100 & 7,852 & $4,207,546$ & 43.0 & 99.8 & 46.7 & 0.44 \\
\hline Male & 2,839 & 3,707 & 2,928 & $1,640,249$ & 43.4 & 99.8 & 49.2 & 0.46 \\
\hline Female & 4,033 & 5,393 & 4,924 & $2,567,297$ & 42.8 & 99.8 & 45.0 & 0.44 \\
\hline
\end{tabular}

Abbreviations: AAPI $=$ Asian American/Pacific Islanders/Native Hawaiians; AIAN $=$ American Indians/Alaskan Natives. 
TABLE 2 Beneficiaries with race misclassified by EDB or RTI compared to OASIS;

$$
\mathrm{N}=4,231,370
$$

\section{OASIS race}

\begin{tabular}{lcrrrrr}
\hline \multicolumn{6}{l}{ EDB race $-318,177(7.5 \%)$} & misclassified \\
& & Hispanic & AAPI & White & Black & AIAN \\
\hline Hispanic & 9,079 & - & 753 & 6,695 & 1,481 & 150 \\
& & & $8.3 \%$ & $73.7 \%$ & $16.3 \%$ & $1.7 \%$ \\
\hline AAPI & 10,462 & 1,124 & - & 7,786 & 728 & 824 \\
& & $10.7 \%$ & & $74.4 \%$ & $7.0 \%$ & $7.9 \%$ \\
\hline White & 190,434 & 167,495 & 8,532 & - & 8,376 & 6,031 \\
& & $88.0 \%$ & $4.5 \%$ & & $4.4 \%$ & $3.2 \%$ \\
\hline Black & 28,042 & 8,167 & 1,155 & 17,524 & - & 1,196 \\
& & $29.1 \%$ & $4.1 \%$ & $62.5 \%$ & & $4.3 \%$ \\
\hline AIAN & 8,061 & 517 & 439 & 6,614 & 491 & - \\
& & $6.4 \%$ & $5.5 \%$ & $82.1 \%$ & $6.1 \%$ & \\
\hline Other/ & 72,099 & 12,749 & 753 & 31,364 & 4,554 & 879 \\
Unknown & & $17.7 \%$ & $8.3 \%$ & $43.5 \%$ & $6.3 \%$ & $1.2 \%$ \\
\hline
\end{tabular}

RTI race $-179,488$ (4.2\%) misclassified

\begin{tabular}{lcrrrrr}
\hline & & Hispanic & AAPI & White & Black & AIAN \\
\hline Hispanic & 48,592 & - & 6,214 & 37,670 & 4,175 & 533 \\
& & & $12.8 \%$ & $77.5 \%$ & $8.6 \%$ & $1.1 \%$ \\
\hline \multirow{2}{*}{ AAPI } & 15,594 & 1,373 & - & 11,948 & 1,160 & 1,113 \\
& & $8.8 \%$ & & $76.6 \%$ & $7.4 \%$ & $7.1 \%$ \\
\hline White & 41,878 & 21,941 & 6,589 & - & 7,682 & 5,666 \\
& & $52.4 \%$ & $15.7 \%$ & & $18.3 \%$ & $13.5 \%$ \\
\hline Black & 21,699 & 2,298 & 960 & 17,265 & - & 1,176 \\
& & $10.6 \%$ & $4.4 \%$ & $80.0 \%$ & & $5.4 \%$ \\
\hline AIAN & 7,852 & 446 & 362 & 6,570 & 474 & - \\
& & $5.7 \%$ & $4.6 \%$ & $83.7 \%$ & $6.0 \%$ & \\
\hline Other/ & 43,873 & 1,377 & 8,448 & 29,203 & 4,233 & 612 \\
Unknown & & $3.1 \%$ & $19.3 \%$ & $66.6 \%$ & $9.6 \%$ & $1.4 \%$ \\
\hline Abbrev & & AAPI & & &
\end{tabular}

Abbreviations: AAPI $=$ Asian American/Pacific Islanders/Native Hawaiians; $\quad$ AIAN $=$ American Indians/Alaskan Natives. 
TABLE 3 Number and ratio/net difference of Medicare beneficiaries from each racial/ethnic group who have a dementia or diabetes flag in the Medicare Chronic Conditions Warehouse using RTI race and EDB race compared to OASIS race.

\begin{tabular}{|c|c|c|c|c|c|c|c|}
\hline \multicolumn{8}{|c|}{ Dementia sample; $\mathrm{n}=1,195,145 / 4,231,370$} \\
\hline & OASIS & RTI & Ratio & Net difference & EDB & Ratio & Net difference \\
\hline Hispanic & 88,272 & 92,555 & 1.05 & $+4.9 \%$ & 39,865 & 0.45 & $-54.8 \%$ \\
\hline AAPI & 28,653 & 26,800 & 0.94 & $-6.4 \%$ & 22,621 & 0.79 & $-21.1 \%$ \\
\hline White & 932,097 & 918,955 & 0.99 & $-1.4 \%$ & 966,390 & 1.04 & $+3.7 \%$ \\
\hline Black & 142,112 & 143,526 & 1.01 & $+1.0 \%$ & 145,651 & 1.01 & $+1.5 \%$ \\
\hline AIAN & 4,011 & 3,662 & 0.91 & $-8.7 \%$ & 3,721 & 0.93 & $-7.2 \%$ \\
\hline Other/Unknown & & 9,647 & & & 16,897 & & \\
\hline \multicolumn{8}{|c|}{ Diabetes sample; $\mathrm{n}=2,018,686 / 4,231,370$} \\
\hline & OASIS & RTI & Ratio & Net difference & EDB & Ratio & Net difference \\
\hline Hispanic & 193,274 & 203,751 & 1.05 & $+5.4 \%$ & 79,271 & 0.41 & $-59.0 \%$ \\
\hline AAPI & 53,705 & 49,314 & 0.92 & $-8.2 \%$ & 41,592 & 0.77 & $-22.6 \%$ \\
\hline White & $1,435,215$ & $1,406,189$ & 0.98 & $-2.0 \%$ & $1,516,959$ & 1.06 & $+5.7 \%$ \\
\hline Black & 326,941 & 329,158 & 1.01 & $+0.8 \%$ & 334,490 & 1.02 & $+2.3 \%$ \\
\hline AIAN & 9,551 & 8,902 & 0.93 & $-6.8 \%$ & 9,050 & 0.95 & $-5.2 \%$ \\
\hline Other/Unknown & & 9,647 & & & 37,324 & & \\
\hline
\end{tabular}

Abbreviations: AAPI = Asian American/Pacific Islanders/Native Hawaiians; AIAN = American Indians/Alaskan Natives. Note: Denominator for prevalence of chronic illness is based on the racial/ethnic subgroup population specific to each race variable (OASIS, EDB, RTI). 
TABLE 4 Comparison of accuracy and agreement measures for EDB and RTI race with prior studies.

\begin{tabular}{|c|c|c|c|c|c|c|c|c|c|c|c|c|}
\hline & \multicolumn{4}{|c|}{$\begin{array}{l}\text { Waldo } \\
\text { EDB 1998-2001 }(\mathrm{n}=32,038) \\
\text { Reference: MCBS 1998-2001 }\end{array}$} & \multicolumn{4}{|c|}{$\begin{array}{c}\text { Eicheldinger \& Bonito } \\
\text { EDB } 2003(\mathrm{n}=830,728) \\
\text { Reference: CAHPS 2000-2002 }\end{array}$} & \multicolumn{4}{|c|}{$\begin{array}{l}\text { [Authors - this paper] } \\
\text { EDB } 2015(\mathrm{n}=4,231,370) \\
\text { Reference: OASIS } 2015\end{array}$} \\
\hline & Sensitivity & Specificity & PPV & Kappa & Sensitivity & Specificity & PPV & Kappa & Sensitivity & Specificity & PPV & Kappa \\
\hline Hispanic & 35.7 & 99.9 & 97.5 & 0.50 & 29.5 & 99.9 & 92.7 & 0.43 & 36.2 & 99.8 & 92.2 & 0.50 \\
\hline$A A P I$ & 54.0 & 99.8 & 70.0 & 0.61 & 54.7 & 99.8 & 84.5 & 0.66 & 65.0 & 99.7 & 79.3 & 0.71 \\
\hline White & 96.5 & 88.2 & 98.2 & 0.81 & 99.3 & 61.7 & 91.7 & 0.71 & 97.9 & 79.5 & 94.4 & 0.81 \\
\hline Black & 95.6 & 99.6 & 96.5 & 0.96 & 97.4 & 98.8 & 86.3 & 0.91 & 97.0 & 99.2 & 94.8 & 0.95 \\
\hline \multirow[t]{8}{*}{$A I A N$} & 20.6 & 99.9 & 69.5 & 0.32 & 35.7 & 99.9 & 59.9 & 0.45 & 43.1 & 99.8 & 46.1 & 0.44 \\
\hline & & & & & \multicolumn{4}{|c|}{$\begin{array}{l}\text { Bonito, Bann, Eicheldinger, et al. } \\
\text { RTI NEWRACE } 2003(\mathrm{n}=830,728) \\
\text { Reference: CAHPS 2000-2002 }\end{array}$} & \multicolumn{4}{|c|}{$\begin{array}{c}\text { [Authors - this paper] } \\
\text { RTI } 2015(\mathrm{n}=4,231,370) \\
\text { Reference: OASIS } 2015\end{array}$} \\
\hline & & & & & Sensitivity & Specificity & PPV & Kappa & Sensitivity & Specificity & PPV & Kappa \\
\hline & & & & Hispanic & 76.6 & 99.2 & 84.5 & 0.79 & 90.8 & 98.7 & 84.8 & 0.87 \\
\hline & & & & $A A P I$ & 79.2 & 99.7 & 81.5 & 0.80 & 74.7 & 99.6 & 81.0 & 0.77 \\
\hline & & & & White & - & - & - & - & 96.9 & 95.5 & 98.7 & 0.90 \\
\hline & & & & Black & - & - & - & - & 96.6 & 99.4 & 95.9 & 0.96 \\
\hline & & & & $A I A N$ & - & - & - & - & 43.0 & 99.8 & 46.7 & 0.44 \\
\hline
\end{tabular}

Abbreviations: AAPI = Pacific Islanders; AIAN = American Indians/Alaskan Natives. 